\title{
Relationship Between Prehospital Time and 24-h Mortality in Road Traffic-Injured Patients in Laos
}

\author{
Takaaki Suzuki $^{1,2} \cdot$ Oulaivanh Phonesavanh $^{3} \cdot$ Snong Thongsna $^{4} \cdot$ Yoshiaki Inoue $^{2}$. \\ Masao Ichikawa ${ }^{1}$
}

Accepted: 22 December 2021/Published online: 18 January 2022

(C) The Author(s) 2022

\begin{abstract}
Background Road traffic injury has long been regarded as a "time-dependent disease." However, shortening the prehospital time might not improve the outcome in developing countries given the current quality of in-hospital care. We aimed to examine the relationship between the prehospital time and 24-h mortality among road traffic victims in Laos.

Methods A prospective observational study was conducted using the trauma registry data on traffic-injured patients who were transported by ambulance to a trauma center in the capital city of Laos from May 2018 to April 2019. The analysis focused on patients with non-mild conditions, whose outcomes could be affected by the prehospital time. To examine the relationship between a prehospital time of $<60 \mathrm{~min}$ and 24-h mortality, a generalized estimating equation model was used incorporating the inverse probability weights utilizing the propensity score for the prehospital time.

Results Of 701 patients, $73 \%$ were men, $91 \%$ were riding 2- or 3-wheel motor vehicles during the crash, and $68 \%$ had a prehospital time of $<60 \mathrm{~min}$. A total of 35 patients died within $24 \mathrm{~h}$ after the crash. Compared with those who survived, individuals who died tended to have head and torso injuries. The proportions of 24-h mortality were $4.7 \%$ and $5.4 \%$ in patients whose prehospital time was $<60 \mathrm{~min}$ and $\geq 60 \mathrm{~min}$, respectively. No significant relationship was found between the prehospital time and 24-h mortality.

Conclusion A shorter prehospital time was not associated with the 24-h survival among road traffic victims in Laos.
\end{abstract}

Takaaki Suzuki

takasuzuki@md.tsukuba.ac.jp

1 Department of Global Public Health, Faculty of Medicine, University of Tsukuba, 1-1-1 Tennodai, Tsukuba, Ibaraki 305-8575, Japan

2 Department of Emergency and Critical Care Medicine, Faculty of Medicine, University of Tsukuba, 1-1-1 Tennodai, Tsukuba, Ibaraki 305-8575, Japan

3 Emergency Department, Mittaphab Hospital, Ministry of Health, Vientiane, Lao People's Democratic Republic

4 Ministry of Health, Vientiane, Lao People's Democratic Republic

\section{Introduction}

Road traffic injuries have long been regarded as a "timedependent disease." Hospital arrival within an hour after the injury (the so-called "golden hour") can improve the patient's health outcomes [1-9]. Currently, minimizing the time interval between traffic crashes and the provision of first professional emergency care is one of the global road safety performance targets [10]. To achieve this target, an emergency medical service (EMS) system must be developed. However, many developing countries lack EMS systems, and traffic deaths prior to hospital arrival are partly attributable to the lack of timely post-crash responses and access to hospitals [11-14]. 
The World Health Organization advocates the establishment of EMS systems in developing countries [12]; however, shortening of the prehospital time might not improve the outcome given the current quality of in-hospital care. In developing countries, the hospital facilities may not be fully equipped; additionally, the healthcare workers may not have the necessary skills to resuscitate severely injured patients with head injuries and torso injuries with major bleeding [14-18], which are two major causes of trauma-related death in the early phase. Therefore, the effect of shortening the prehospital time could be less than expected, unless both the quality of in-hospital care and prehospital care is improved.

The relationship between the prehospital time and outcome has been examined mainly in developed countries [1-9, 19-29]; several studies have reported that the shorter the prehospital time, the better the outcome [30, 31]. However, it remains unclear whether this finding is applicable to developing countries, especially in low-resource settings, because of the current quality of in-hospital care.

Conversely, previous studies have two major limitations in estimating the effect of prehospital time on the outcome. First, patients with mild conditions whose outcomes were not affected by the prehospital time were included in the analyses. If the prehospital time of these patients was prolonged because they were not in urgent condition, the impact of the prehospital time might have been underestimated, especially when the patients with mild conditions comprised the majority of the study population. Therefore, it is reasonable to exclude them.

Second, the factors influencing the outcomes, namely vital signs, were inappropriately adjusted in the analyses. Generally, the vital signs measured upon hospital arrival are adjusted in analysis; however, this adjustment might have underestimated the effect of the prehospital time on the outcomes because the possible aggravation of vital signs from the scene to the hospital (due to prehospital time) was cancelled out due to the adjustment. To estimate the effect of the prehospital time on the outcomes, the analysis should control for vital signs measured at the scene.

In Laos, one of the 46 least developed countries, traffic injury is currently one of the main causes of total death [32]. The number of traffic-related deaths per 100,000 people has nearly doubled in the past decade; of the 7.3 million people living in Laos, 1,031 traffic victims died in 2020 [10, 33]. Rescue teams with ambulances have been voluntarily formed by citizens to respond to a rapid increase in traffic victims, and the EMS system has been informally established in the capital city. This situation allowed the examination of whether shortening the prehospital time is beneficial in the context of low-resource settings. Therefore, this study aimed to examine the relationship between a prehospital time of $<60 \mathrm{~min}$ and the 24-h mortality among traffic victims transported by ambulance in Laos. To overcome the limitations of previous studies, our analysis focused on victims with non-mild conditions, and vital signs at the scene were adjusted.

\section{Materials and methods}

\section{Study setting and participants}

A prospective observational study was conducted in traffic victims transported by ambulance from the scene to Mittaphab Hospital in Vientiane Capital, the capital city of Laos, from May 1, 2018, to April 30, 2019. The patients transported by non-ambulance vehicles were excluded because their condition was mostly mild, and their mortality risk would not be affected by the prehospital time. In fact, nearly all such patients survived. In Laos, this hospital is the only trauma center, and traffic victims are largely motorcycle riders as motorcycles are the primary mode of transportation. To examine the association of prehospital time with mortality, patients transferred from other hospitals were excluded. Patients who experienced cardiac pulmonary arrest (CPA) at the scene were also excluded because they hardly survived and their shock index (SI) could not be calculated, while patients who experienced CPA on the way to the hospital were included.

\section{EMS in Laos}

During the study period, the EMS consisted of 7 rescue teams with assigned emergency call numbers, 34 ambulances, and 650 crew members at Vientiane Capital, which has a population of nearly 0.7 million people and an area of $3920 \mathrm{~km}^{2}$. All rescue teams are trained to carry out primary trauma care, such as basic life support, cervical spine protection, manual airway management and pressure hemostasis. Occasionally, the ambulance vehicles without equipment for measuring the vital signs were used to reach the scene, resulting in missing data on vital signs; however, this happened most likely at random because the ambulance vehicles were not systematically dispatched.

Almost all patients with severe trauma were either directly transported from the scene or transferred from other hospitals to Mittaphab Hospital. There were no certified emergency physicians, and the international standard resuscitation was not able to be practiced. Massive transfusion cannot be performed in patients with shock. Emergency surgery could be performed if the operating rooms were not occupied. 


\section{Data}

Anonymous and unconsolidated data on road traffic-injured patients were obtained from the trauma registry at Mittaphab Hospital, which was established in 2018. The trauma registry consisted of prehospital records used in the ambulance and medical records used by the hospital. The prehospital records were filled by rescue teams concurrently with patient care, and included the basic patient information and data on the time course, vital signs, injuries, and treatment. The hospital staff was responsible for collecting and registering the data. The data were primarily recorded in the paper-based form and then registered electronically.

The following data were collected: age and sex; time of ambulance dispatch, arrival at the scene, leaving the scene, and arrival at the hospital; name of the rescue team; type of vehicle used by the patient and by the person collided with; prehospital and in-hospital vital signs (heart rate, systolic blood pressure, consciousness level based on the Glasgow Coma Scale [GCS]) measured at the scene and hospital arrival, respectively; injured body part (head, face, neck, chest, abdomen, spine, upper limbs, and lower limbs); outcome (discharged to home, transferred to another hospital, and died); and date of discharge, transfer, or death.

\section{Measures}

The exposure and outcome variables were prehospital time (time interval from ambulance dispatch to arrival at the hospital) and 24-h mortality. The patients who were discharged or transferred to another hospital within $24 \mathrm{~h}$ were considered as survivors. The patients' condition was determined as mild or non-mild based on their prehospital SI and GCS scores, as shown in Table 1. To measure the anatomical severity, the number of seriously injured body parts with more than three points on the Abbreviated Injury Scale (AIS) were determined. However, the Injury Severity

Table 1 Severity of condition based on the prehospital shock index and Glasgow Coma Scale score

\begin{tabular}{lll}
\hline & \multicolumn{2}{l}{ Prehospital Glasgow Coma Scale score } \\
\cline { 2 - 3 } & Less than 13 & 13 or more \\
\hline Prehospital shock index & & \\
1 or more & Non-mild & Non-mild \\
Less than 1 & Non-mild & Mild
\end{tabular}

${ }^{a}$ Based on the vital signs measured at the scene

${ }^{b}$ Shock index was calculated by dividing the heart rate by the systolic blood pressure

${ }^{\mathrm{c}}$ Glasgow Coma Scale score range from 3 to 15
Score was not calculated as the six-point AIS coding could not be performed.

\section{Analysis}

The analysis focused on patients with non-mild conditions for the reasons stated earlier. Prior to the analysis, the patients whose outcomes were unknown were excluded, and any missing values of the variables were supplemented using the multiple imputation method, which included the response time (from ambulance dispatch to arrival at the scene), on-scene time (from arrival at the scene to leaving the scene), transport time (from leaving the scene to arrival at the hospital), and prehospital systolic blood pressure, heart rate, and the GCS score. Considering the amount of missing data, 50 pseudo-complete datasets were created through imputation [34]. Subsequently, the characteristics of the patients with non-mild conditions were compared between those who died within $24 \mathrm{~h}$ after the crash and those who survived. This comparison was made using the Wilcoxon rank sum test, Kruskal-Wallis test, or Pearson's chi-square test, depending on the type and distribution of the variable. Rubin's rules were used to average across the imputed datasets.

Finally, the relationship between a prehospital time of $<60 \mathrm{~min}$ and 24-h mortality was examined. First, the propensity score for a prehospital time of $<60$ min was calculated because the probability of patients having a prehospital time of $<60$ min might depend on their condition at the scene, which might confound the relationship. To calculate the propensity score, a logistic regression analysis was conducted with adjustment for covariates, including age, sex, rescue team, prehospital SI and GCS scores, serious injury to each body part, time zone when transported, and type of vehicle used by the patients and by the person collided with (Supplementary Table 1). Subsequently, to estimate the association of a prehospital time of $<60 \mathrm{~min}$ and 24-h mortality, a generalized estimating equation model was used incorporating the inverse probability weights based on the propensity score [35]; the odds ratio (OR) and its $95 \%$ confidence interval (CI) were provided. The analysis was performed using SPSS version 27.0 (IBM Corp., Armonk, NY, USA).

\section{Results}

A total of 18,995 patients involved in road traffic crashes visited the Mittaphab Hospital during the study period and 5,604 patients of them were transported by ambulance. Of these, 1,455 patients were transported from other hospitals, 18 patients experienced CPA at the scene, and 8 patients had undocumented outcomes. These patients were 
excluded from the analysis. A total of 4,123 patients were included in the analyses for missing data imputation. Supplementary Table 2 shows the patient characteristics before the imputation. Additionally, the patients were classified into those with mild or non-mild conditions based on the prehospital SI and GCS scores.

Table 2 shows the characteristics of 701 patients with non-mild conditions, including those who died within $24 \mathrm{~h}$
( $n=35)$ and those who survived $(n=666)$. Of the patients with a median age of 25 years, $73 \%$ were men, $91 \%$ were driving or riding 2- or 3-wheel motor vehicles during the crash, and $44 \%$ were transported by Vientiane Rescue 1623, the first rescue team established in Laos with the largest number of members and equipment. The median response time, on-scene time, and transport time were 12 , 7, and $16 \mathrm{~min}$, respectively, and 475 patients had a

Table 2 Characteristics of traffic-injured patients with non-mild conditions who were transported to Mittaphab Hospital by ambulance after a road traffic crash from May 2018 to April 2019 and the 24-h mortality rates ${ }^{\mathrm{a}}$

\begin{tabular}{|c|c|c|}
\hline & Died $(n=35)$ & Survived $(n=666)$ \\
\hline Age (years) & $23[19,39]$ & $25[21,35]$ \\
\hline Sex (male) & $26(74 \%)$ & $489(73 \%)$ \\
\hline \multicolumn{3}{|l|}{ Type of vehicle used by the patients } \\
\hline Pedestrian & $0(0 \%)$ & $22(3.3 \%)$ \\
\hline Bicycle & $0(0 \%)$ & $4(0.6 \%)$ \\
\hline 2- or 3-wheel & $33(94 \%)$ & $606(91 \%)$ \\
\hline 4-wheel & $2(5.7 \%)$ & $32(4.8 \%)$ \\
\hline Rescue team (Vientiane Rescue 1623) & $15(45 \%)$ & $293(44 \%)$ \\
\hline \multicolumn{3}{|l|}{ Prehospital time } \\
\hline Response time $^{\mathrm{b}}$ & $10[5,32]$ & $11[5,26]$ \\
\hline On-scene time ${ }^{c}$ & $7[5,9]$ & $8[5,13]$ \\
\hline Transport time $^{\mathrm{d}}$ & $13[9,35]$ & $20[10,37]$ \\
\hline Prehospital time (less than $60 \mathrm{~min}$ ) & $22(63 \%)$ & $453(68 \%)$ \\
\hline \multicolumn{3}{|l|}{ Prehospital vital signs ${ }^{\mathrm{e}}$} \\
\hline Shock index & $0.8[0.7,0.9]$ & $0.8[0.7,1.0]$ \\
\hline Glasgow Coma Scale & $3[3,3]$ & $6[5,7]$ \\
\hline \multicolumn{3}{|l|}{ In-hospital vital signs ${ }^{\mathrm{f}}$} \\
\hline Shock index & $0.9\left[\begin{array}{ll}0.5 & 1.3\end{array}\right]$ & $0.8[0.7,0.9]$ \\
\hline Glasgow Coma Scale & $3[3,4]$ & $12[7,15]$ \\
\hline \multicolumn{3}{|l|}{ Serious injury to each body part ${ }^{\mathrm{g}}$} \\
\hline Head & $32(91 \%)$ & $175(26 \%)$ \\
\hline Face & $21(60 \%)$ & $158(24 \%)$ \\
\hline Neck & $7(20 \%)$ & $5(0.8 \%)$ \\
\hline Chest & $17(49 \%)$ & $29(4.4 \%)$ \\
\hline Abdomen & $12(34 \%)$ & $26(3.9 \%)$ \\
\hline Spine & $0(0 \%)$ & $2(0.3 \%)$ \\
\hline Upper limbs & $4(11 \%)$ & $67(10 \%)$ \\
\hline Lower limbs & $13(37 \%)$ & $140(21 \%)$ \\
\hline Number of seriously injured body parts ${ }^{h}$ & $3[2,4]$ & $2[1,2]$ \\
\hline
\end{tabular}

${ }^{\mathrm{a}}$ Values other than the frequency $(\%)$ are expressed as median [interquartile range]

${ }^{\mathrm{b}}$ From ambulance dispatch to arrival at the scene

${ }^{\mathrm{c}}$ From arrival at the scene to leaving the scene

${ }^{\mathrm{d}}$ From leaving the scene to hospital arrival

${ }^{\mathrm{e}}$ Measured at the scene

${ }^{\mathrm{f}}$ Measured at hospital arrival

${ }^{\mathrm{g}}$ Injury with more than three points on the Abbreviated Injury Scale (AIS)

${ }^{\mathrm{h}}$ Number of body parts containing injuries with more than three points on the AIS 
prehospital time of less than $60 \mathrm{~min}$. The most common injured parts were the head (30\%), face $(26 \%)$, and lower limbs $(22 \%)$. Compared with those who survived, patients who died were more likely to have injuries in the head, chest, and abdomen.

Table 3 shows the association between the prehospital time and 24-h mortality. The proportion of 24-h mortality was $4.7 \%$ and $5.4 \%$ in patients with a prehospital time of $<60 \mathrm{~min}$ and those with a prehospital time of $60 \mathrm{~min}$ or longer, respectively. No significant association was found between the prehospital time and 24-h mortality.

\section{Discussion}

A prehospital time of $<60$ min was not significantly associated with the 24-h mortality in road traffic-injured patients with non-mild conditions in Laos. To interpret this finding, caution should be exercised because the impact of prehospital time was not precisely estimated due to a small number of outcomes, though potential explanations for the lack of significant association were provided below for future research.

First, hospitals do not have the capacity to provide highquality resuscitation, even if the patients are transported to the hospital within an hour. The quality of in-hospital care provided to trauma patients is not only determined solely by the skills of the surgeons, but also by the diagnostic skills, supportive treatment, and perioperative management. To stabilize shock patients with active bleeding, massive transfusions are required [36]. Even when operations are perfectly conducted, the outcomes depend highly on the quality of anesthetic management and postoperative

Table 3 Proportion of 24-h mortality by prehospital time among traffic-injured patients with non-mild condition who were transported to Mittaphab Hospital by ambulance after a road traffic crash from May 2018 to April 2019, and the association of prehospital time with 24-h mortality

\begin{tabular}{lll}
\hline Prehospital time $^{\mathrm{a}}$ & $\%$ & OR $(95 \% \mathrm{CI})^{\mathrm{b}}$ \\
\hline Less than $60 \mathrm{~min}$ & 4.7 & $0.99(0.42-2.42)$ \\
60 min or more & 5.4 & Reference
\end{tabular}

$O R$ odds ratio, $C I$ confidence interval

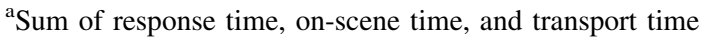

${ }^{b}$ Estimated using the generalized estimating equations model incorporating the inverse probability weights using the propensity score for prehospital time of $<60 \mathrm{~min}$ (based on age, sex, rescue team, prehospital shock index, prehospital Glasgow Coma Scale score, serious injury to each body part with more than three points on the Abbreviated Injury Scale, time zone when transported, and the type of vehicle used by the patients and by the person collided with) care provided in the intensive care unit. Such in-hospital care is not always available in Laos.

Second, even if the time from the injury to hospital arrival was shortened, the time from hospital arrival to resuscitation might not have been shortened. In Laos, the prehospital information is not shared with the hospital before hospital arrival, which delays the formation of trauma teams to resuscitate the patients. Moreover, it is not always possible to conduct emergency operations because of the limited capacity to manage operating rooms. In this case, the patients needed to wait until the ongoing operations were completed, which may be deleterious, especially for patients requiring urgent care.

Third, traffic crashes that occur in suburban areas may be less likely witnessed than those that occur in urban areas. In addition, the emergency call numbers may not be widely known and are less likely to be known by people living in suburban areas, as EMS is not officially provided as a public service. As a result, some emergency calls and ambulance dispatches may be delayed. If this was the case, paradoxically, the outcomes of patients transported from suburban areas with a longer prehospital time could be better than those from urban areas as patients requiring urgent care were more likely to die at the scene before they were noticed in suburban areas, and only those with better condition were transported to the hospital.

This study had four major limitations. First, there was an inherent uncertainty in our estimates due to the large missing data with a small number of outcomes despite the data imputation. In order to deal with the missing data, a large number of imputed datasets were generated for analysis. To consider a large number of potential confounders relative to a small number of outcomes, the propensity score method was used. Yet, the impact of prehospital time should be further precisely estimated in a larger sample size. Second, there might be a potential misclassification of non-mild conditions into mild in patients with severe head or torso injuries, if their prehospital SI and GCS scores indicated mild conditions despite sustaining severe injuries. These cases were not included in the analysis. This might have led to the underestimation of the relationship between prehospital time and mortality. However, this could be occurred only if there were many such cases and the prehospital time was shortened due to the presence of head or torso injuries. Third, there was a paucity of information, such as the time from the crash to ambulance dispatch and the location of the crash, which might help explain the lack of association. Fourth, our findings are not applicable to other low-resource settings that employ a "stay-and-play" type of EMS, unlike the "scoop-and-run" type, which exists in Laos. In the "stay-and-play" type, on-scene treatment and resuscitation, such as endotracheal intubation and blood 
transfusion at the scene, are prioritized over rapid transportation [37-39].

In conclusion, the prehospital time was not significantly associated with 24-h mortality among traffic-injured patients with non-mild conditions, transported by ambulance in Laos. The quality of prehospital and in-hospital care that might affect the survival should be evaluated further in a larger sample size to estimate the impact of prehospital time more precisely.

Supplementary Information The online version contains supplementary material available at https://doi.org/10.1007/s00268022-06445-9.

Acknowledgements The authors are very thankful to Drs. Tavang Manivong and Vangnakhone Dittaphong for their technical supports.

Funding This work was partially supported by the Foundation for Advanced Studies on International Development Scholarship Program, Japan.

\section{Declarations}

Conflict of interest The authors declare that they have no conflicts of interest.

Informed consent Informed consent for participation could not be obtained from all individual participants owing to the retrospective nature of the present study. Instead, the study protocol and supporting contents are published on the webpage of the University of Tsukuba Hospital. The opportunity and rights of patients to opt out of the study were guaranteed.

Ethical approval The study protocol was approved by the Institutional Review Board of the University of Tsukuba Hospital, and the study was conducted in accordance with the Declaration of Helsinki.

Open Access This article is licensed under a Creative Commons Attribution 4.0 International License, which permits use, sharing, adaptation, distribution and reproduction in any medium or format, as long as you give appropriate credit to the original author(s) and the source, provide a link to the Creative Commons licence, and indicate if changes were made. The images or other third party material in this article are included in the article's Creative Commons licence, unless indicated otherwise in a credit line to the material. If material is not included in the article's Creative Commons licence and your intended use is not permitted by statutory regulation or exceeds the permitted use, you will need to obtain permission directly from the copyright holder. To view a copy of this licence, visit http://creativecommons. org/licenses/by/4.0/.

\section{References}

1. Dinh MM, Bein K, Roncal S et al (2013) Redefining the golden hour for severe head injury in an urban setting: the effect of prehospital arrival times on patient outcomes. Injury 44:606-610

2. Newgard CD, Meier EN, Bulger EM et al (2015) Revisiting the "golden hour": an evaluation of out-of-hospital time in shock and traumatic brain injury. Ann Emerg Med 66:30-41
3. Kotwal RS, Howard JT, Orman JA et al (2016) The effect of a golden hour policy on the morbidity and mortality of combat casualties. JAMA Surg 151:15-24

4. Esmaeili RA, Mayel M, Movahedi M et al (2016) Pre-hospital time intervals in trauma patient transportation by emergency medical service: association with the first 24-h mortality. J Emerg Pract Trauma 2:37-41

5. Bagher A, Todorova L, Andersson L et al (2017) Analysis of prehospital rescue times on mortality in trauma patients in a Scandinavian urban setting. Trauma 19:28-34

6. Pham H, Puckett Y, Dissanaike S (2017) Faster on-scene times associated with decreased mortality in helicopter emergency medical services (HEMS) transported trauma patients. Trauma Surg Acute Care Open 2:e000122

7. Ruelas OS, Tschautscher CF, Lohse CM et al (2018) Analysis of prehospital scene times and interventions on mortality outcomes in a national cohort of penetrating and blunt trauma patients. Prehosp Emerg Care 22:691-697

8. Chen X, Guyette FX, Peitzman AB et al (2019) Identifying patients with time-sensitive injuries: association of mortality with increasing prehospital time. J Trauma Acute Care Surg 86:1015-1022

9. Tansley G, Schuurman N, Bowes M et al (2019) Effect of predicted travel time to trauma care on mortality in major trauma patients in Nova Scotia. Can J Surg 62:123-130

10. World Health Organization. Global Plan for the Decade of Action for Road Safety 2021-2030. https://www.who.int/publications/m/ item/global-plan-for-the-decade-of-action-for-road-safety-20212030. Accessed December 1st, 2021

11. World Health Organization. Global status report on road safety 2018. https://www.who.int/publications-detail/global-statusreport-on-road-safety-2018. Accessed December 1st, 2021

12. World Health Organization. Post-crash response: Supporting those affected by road traffic crashes. https://www.who.int/pub lications-detail/post-crash-response-supporting-those-affectedby-road-traffic-crashes. Accessed December 1st, 2021

13. Nielsen K, Mock C, Joshipura M et al (2012) Assessment of the status of prehospital care in 13 low- and middle-income countries. Prehosp Emerg Care 16:381-389

14. Reynolds TA, Stewart B, Drewett I et al (2017) The impact of trauma care systems in low- and middle- income countries. Annu Rev Public Health 38:507-532

15. Hashmi ZG, Haider AH, Zafar SN et al (2013) Hospital-based trauma quality improvement initiatives: first step toward improving trauma outcomes in the developing world. J Trauma Acute Care Surg 75:60-68

16. Whitaker J, Denning M, O’Donohoe N et al (2019) Assessing trauma care health systems in low- and middle-income countries, a protocol for a systematic literature review and narrative synthesis. Syst Rev 8:157

17. Pittalis C, Brugha R, Gajewski J (2019) Surgical referral systems in low- and middle-income countries: a review of the evidence. PLoS ONE 14:e0223328

18. Selveindran SM, Tango T, Khan MM et al (2019) Mapping global evidence on strategies and interventions in neurotrauma and road traffic collisions prevention: a scoping review protocol. BMJ Open 9:e031517

19. Alexander FB, Lina VM, Chelsea D et al (2020) A scoping review of worldwide studies evaluating the effects of prehospital time on trauma outcomes. Int J Emerg Med 13(1):64

20. Rogers FB, Rittenhouse KJ, Gross BW (2015) The golden hour in trauma: dogma or medical folklore? Injury 46:525-527

21. Newgard CD, Schmicker RH, Hedges JR et al (2010) Emergency medical services intervals and survival in trauma: assessment of the "golden hour" in a North American prospective cohort. Ann Emerg Med 55(235-546):21 
22. McCoy CE, Menchine M, Sampson S et al (2013) Emergency medical services out-of-hospital scene and transport times and their association with mortality in trauma patients presenting to an urban Level I trauma center. Ann Emerg Med 61:167-174

23. Dharap SB, Kamath S, Kumar V (2017) Does prehospital time affect survival of major trauma patients where there is no prehospital care? J Postgrad Med 63:169-175

24. Clements TW, Vogt K, Hameed SM et al (2017) Does increased prehospital time lead to a "Trial of Life" effect for patients with blunt trauma? J Surg Res 216:103-108

25. Möller A, Hunter L, Kurland L et al (2018) The association Between hospital arrival time, transport method, prehospital time intervals, and in-hospital mortality in trauma patients presenting to Khayelitsha Hospital, Cape Town. Afr J Emerg Med 8:89-94

26. Brown E, Tohira H, Bailey P et al (2019) Longer prehospital time was not associated with mortality in major trauma: a retrospective cohort study. Prehosp Emerg Care 23:527-537

27. Mills EHA, Aasbjerg K, Hansen SM et al (2019) Prehospital time and mortality in patients requiring a highest priority emergency medical response: a Danish Registry-Based Cohort Study. BMJ Open 9:e023049

28. Klein K, Lefering R, Jungbluth $P$ et al (2019) Is prehospital time important for the treatment of severely injured patients? A matched-triplet analysis of 13,851 patients from the trauma register DGUß. Biomed Res Int 2019:5936345

29. Jungeun K, Kyoung JS, Sang DS et al (2017) Does prehospital time influence clinical outcomes in severe trauma patients? A cross sectional study. Prehosp Emerg Care 21:466-475

30. Harmsen AM, Giannakopoulos GF, Moerbeek PR et al (2015) The influence of prehospital time on trauma patients outcome: a systematic review. Injury 46:602-609
31. María JG, Bryam J (2020) Association between pre-hospital care time and hospital mortality in victims of traffic accidents. Rev Fac Med Hum 20:144-152

32. Phoummalaysith B, Louangpradith V, Manivon T et al (2017) Underlying cause of death recorded during 2013-2015 at a tertiary general hospital in Vientiane Capital, Lao PDR. Nagoya J Med Sci 79:199-209

33. Lao Statistical Information Service Web site. https://laosis.lsb. gov.la. Accessed December 1st, 2021

34. von Hippel PT (2020) How many imputations do you need? A two-stage calculation using a quadratic rule. Sociological Methods \& Research 49:699-718

35. Austin PC (2011) An introduction to propensity score methods for reducing the effects of confounding in observational studies. Multivariate Behav Res 46:399-424

36. Lim G, Harper-Kirksey K, Parekh R et al (2018) Efficacy of a massive transfusion protocol for hemorrhagic trauma resuscitation. Am J Emerg Med 36:1178-1181

37. Smith RM, Conn AK (2009) Prehospital care-scoop and run or stay and play? Injury 40:23-26

38. Wyen H, Lefering R, Maegele M et al (2013) The golden hour of shock-how time is running out: prehospital time intervals in Germany-a multivariate analysis of 15,103 patients from the TraumaRegister DGU(R). Emerg Med J 30:1048-1055

39. Gauss T, Ageron FX, Devaud ML et al (2019) Association of prehospital time to in-hospital trauma mortality in a physicianstaffed emergency medicine system. JAMA Surg 154:1117-1124

Publisher's Note Springer Nature remains neutral with regard to jurisdictional claims in published maps and institutional affiliations. 